\title{
Adipose tissue inflammation: are small or large fat cells to blame?
}

\author{
H. Hauner
}

Received: 25 August 2009 / Accepted: 29 October 2009 / Published online: 24 November 2009

(C) Springer-Verlag 2009

Keywords Adipocyte - Adipose differentiation $\cdot$ Fat cell size $\cdot$ Immune cells $\cdot$ Inflammation $\cdot$ Insulin resistance . Obesity

Although adipose tissue is one of the largest organs-and indeed in some individuals the largest-it is still a poorly understood part of the human body. Recognised as an independent organ 80 years ago, in the following decades adipose tissue was only considered to act as a passive organ that serves as an energy store for excess energy, which is readily mobilised under the control of external signals, such as circulating hormones, in the case of energy demand in response to either increased expenditure or food shortage.

This unspectacular perspective changed dramatically only 15 years ago, when two major discoveries were made. The first was that adipose tissue of obese animals and humans produces elevated amounts of TNF- $\alpha[1,2]$. The second, even more important discovery, was the identification of leptin, which was found to act as a classic secreted hormone signalling the energy reserves to the brain and controlling satiety [3]. These sensational observations revolutionised our view of adipose tissue in the subsequent years and stimulated extensive research activity. Among many resulting findings, the observation that adipose tissue in obese individuals is characterised by chronic inflammation is particularly noteworthy [4]. The first immune cells to be described in adipose tissue were macrophages, which belong to the innate

\section{H. Hauner $(\bowtie)$}

Else Kröner Fresenius Centre for Nutritional Medicine,

Technical University of Munich,

Gregor-Mendel-Strasse 2,

85350 Freising-Weihenstephan, Germany

e-mail: hauner@wzw.tum.de immune system and are known to augment inflammation. A positive association was reported between BMI of donors and mean fat cell size and the proportion of macrophages, using specific markers for immunohistochemical detection [4]. There is also growing evidence that other immune cells such a $\mathrm{T}$ lymphocytes infiltrate adipose tissue [5] and may even precede macrophage invasion [6].

Recent studies have focused on the potential role of adipose tissue cellularity in the context of inflammation to search for the cellular components involved in this process, as well as the underlying regulatory mechanisms. So far this work has suggested that more or less all cellular fractions contribute to inflammation, including the enlarged, hypertrophic fat cells that exhibit a shift towards a more proinflammatory state. The various cell types resident in adipose tissue obviously do not exhibit the same expression pattern, but many discrepant results are most probably caused by differences in models and methods. One study reported that very large fat cells, in comparison with normal-size fat cells, show a much higher expression of pro-inflammatory factors, including a variety of chemoattractants which may direct immune cells into adipose tissue, whereas 'protective' and 'anti-inflammatory' factors such as adiponectin and IL-10 are produced at reduced levels [7]. Although the causes and mechanisms mediating this shift are largely unknown, the recently described development of hypoxia under conditions of fat cell hypertrophy was hypothesised to be a potential trigger for the elevated production of chemoattractants and subsequent invasion of immune cells [8]. Furthermore, signals from pre-adipose rather than adipose cells in the hypoxic state may activate proinflammatory pathways in surrounding endothelial cells, which may further augment adipose tissue inflammation [9]. 
The paper by McLaughlin and colleagues [10] published in this issue of Diabetologia provides a new and surprising finding that may further increase our view of the complexity of adipose tissue inflammation. The authors draw attention to the role of fat cell size in human subcutaneous adipose tissue biopsies, focusing on the bimodal size distribution. The statistical analyses performed showed that the mean diameter of the mature adipose cells was not associated with the expression of selected inflammatory genes in the whole tissue. In contrast, the fraction of small adipose cells was consistently associated with inflammatory gene expression, independently of BMI and insulin resistance. The authors speculate that the observed association between the percentage of small fat cells and inflammation may reflect an impairment of adipogenesis and/or terminal differentiation, as they had reported a negative relationship between the proportion of small fat cells and the expression of genes encoding markers of adipose cell differentiation in a previous study [11].

This is a very interesting observation, but is opposite to current thinking. However, some methodological considerations have to be discussed and may help to resolve this obvious contradiction. The authors used the old method of osmium tetroxide fixation of fat cells, in contrast to collagenase digestion and filtration of adipose tissue samples, which is the most frequently used method. With the latter technique, however, most small fat cells are probably lost, e.g. owing to a high specific density or other reasons, and are generally ignored in studies of adipose tissue biology. At the same time, collagenase digestion may also damage particularly the hypertrophic fat cells, resulting in substantial cell breakage. Thus, because of the rapid fixation of all fat cells, the osmium tetroxide method may better reflect the true fat cell size distribution pattern.

A biphasic adipocyte distribution pattern had already been observed in an older, but still important study, using the osmium tetroxide method and scanning electron microscopy for cell sizing [12]. In this study, a very small fat cell population with a mean diameter between 20 and $28 \mu \mathrm{m}$ was identified that was not seen in suspensions of collagenasedigested adipose tissue biopsies, which showed only large mature fat cells. The very small fat cells represented $21-26 \%$ of total fat cell numbers in lean and both moderately and massively obese donors. However, this very low fat cell fraction remained constant in size and proportion, independently of BMI. The authors speculated that these very small fat cells are not simply immature fat cells, but may represent a separate population that may exert other, so far unknown, biological functions in adipose tissue [12].

Although such data are really fascinating for fat cell researchers, some caveats have to be taken into account. One has to keep in mind that the study of McLaughlin et al. was cross-sectional, simply showing associations, and we know little about the dynamic changes that may occur in adipose tissue in vivo. Another limitation of the study affects the selection of inflammatory markers. The authors chose a small panel with CD14 and CD45 as those most closely associated with the fraction of small adipose cells. This may raise additional questions, as CD14 and CD45 are well-known markers of monocytes/macrophages, which also reside in adipose tissue in significant numbers. These markers were reported to be particularly expressed in the stromal cell fraction of adipose tissue, which not only contains resident cells of the monocyte lineage but also the adipocyte precursor cells as the major component, as well as other cell types. Recent studies clearly indicated that the stromal non-fat cell fraction has a much higher proinflammatory activity than the fat cell pool [13]. However, even differentiating fat cells were found to express CD14 and CD68 [14]. Thus, there may be a lot of overlap concerning the expression of inflammatory proteins among the various cell types present in adipose tissue, as already noted [15].

Unfortunately, the gene expression data collected in the study of McLaughlin et al. represent gene expression of the whole adipose tissue samples, thereby not allowing a differentiation of gene expression according to the specific cell fractions. It remains a true challenge for the future to precisely dissect the cellular composition of adipose tissue and to elucidate the distinct biological functions of these various cell types. Many of the current uncertainties and discrepancies across the published literature may simply be because of our lack of selective tools to separate these cellular components.

In conclusion, the observation by McLaughlin and coworkers is a highly interesting and stimulating contribution that will encourage new research to better characterise adipose tissue cellularity and its functional consequences. As suggested by the authors, it would be worthwhile to focus specific attention on this small fat cell fraction and to study its functional role in the context of obesity-linked inflammation and insulin resistance. Thus for the moment, we are still unable to identify the culprit in terms of cellular components responsible for the metabolic disturbances in obesity, but this study adds another piece of knowledge to the puzzle of adipose tissue (dys-)function, until we finally understand this enigmatic organ.

Acknowledgements The research work of the author is funded by the Else Kröner-Fresenius-Foundation, Bad Homburg, Germany.

Duality of interest The author has received honoraria for lectures from sanofi-aventis, Novo-Nordisk, Lilly, GSK, BMS, Novartis and Weight Watchers over the last 5 years.

\section{References}

1. Hotamisligil GS, Shargill NS, Spiegelman BM (1993) Adipose expression of tumor necrosis factor-alpha: direct role in obesitylinked insulin resistance. Science 259:87-91 
2. Hotamisligil GS, Arner P, Caro JF, Atkinson RL, Spiegelman BM (1995) Increased adipose tissue expression of tumor necrosis factor-alpha in human obesity and insulin resistance. J Clin Invest 95:2409-2415

3. Zhang Y, Proenca R, Maffei M et al (1994) Positional cloning of the mouse obese gene and its human homologue. Nature 372:425432

4. Weisberg SP, McCann D, Desai M et al (2003) Obesity is associated with macrophage accumulation in adipose tissue. J Clin Invest 112:1785-1808

5. Bornstein SR, Abu-Asab M, Glasow A et al (2000) Immunohistochemical and ultrastructural localization of leptin and leptin receptor in human white adipose tissue and differentiating human adipose cells in primary culture. Diabetes 49:532-538

6. Kintscher U, Hartge M, Hess K et al (2008) T-lymphocyte infiltration in visceral adipose tissue: a primary event in adipose tissue inflammation and the development of obesity-mediated insulin resistance. Arterioscler Thromb Vasc Biol 28:1304-1310

7. Skurk T, Alberti-Huber C, Herder C, Hauner H (2007) Relationship between adipocyte size and adipokine expression and secretion. J Clin Endocrinol Metab 92:1023-1033

8. Trayhurn P, Wang B, Wood IS (2008) Hypoxia in adipose tissue: a basis for the dysregulation of white adipose tissue. Br J Nutr 100:227-235
9. Mack I, BelAiba RS, Djordjevic T et al (2009) Functional analyses reveal the greater potency of preadipocytes compared with adipocytes as endothelial cell activator under normoxia, hypoxia, and TNFalpha exposure. Am J Physiol Endocrinol Metab 297:E735-E748

10. McLaughlin T, Deng A, Yee G et al. (2009) Inflammation in subcutaneous adipose tissue: relationship to adipose cell size. Diabetologia. doi:10.1007/s00125-009-1496-3

11. McLaughlin T, Sherman A, Tsao P et al (2007) Enhanced proportion of small adipose cells in insulin-resistant vs insulinsensitive obese individuals implicates impaired adipogenesis. Diabetologia 50:1707-1713

12. Julien P, Despres J-P, Angel A (1989) Scanning electron microscopy of very small fat cells and mature fat cells in human obesity. J Lipid Res 30:293-299

13. Fain JN, Buehrer B, Bahouth SW et al (2008) Comparison of messenger RNA distribution for 60 proteins in fat cells vs the nonfat cells of human omental adipose tissue. Metabolism 57:10051015

14. Khazen W, M'bika JB, Tomkiewicz C et al (2005) Expression of macrophage-selective markers in human and rodent adipocytes. FEBS Lett 579:5631-5634

15. Wellen KE, Hotamisligil GS (2005) Inflammation, stress, and diabetes. J Clin Invest 115:1111-1119 